\title{
A UNIFIED APPROACH TO VORTEX MOTION LAWS OF COMPLEX SCALAR FIELD EQUATIONS
}

\author{
F.-H. LiN AND J. X. XIN
}

\begin{abstract}
In this short note, we give a unified rigorous derivation of vortex motion laws of nonlinear wave (NLW) and nonlinear heat (NLH) equations based on the fluid dynamic approach the authors recently developed in solving the nonlinear Schrödinger (NLS) equation. Hence in all three complex scalar field equations, the motion laws follow from the Euler-type equations, and the knowledge of the finite mass Radon defect measure.
\end{abstract}

\section{A summary of basic facts}

Let us consider as $\epsilon \downarrow 0$ the two-dimensional complex scalar field equations:

$$
\frac{1}{\log \epsilon^{-1}} u_{\epsilon, t t}=\Delta u_{\epsilon}+\epsilon^{-2} u_{\epsilon}\left(1-\left|u_{\epsilon}\right|^{2}\right)
$$

the nonlinear wave (NLW) equation;

$$
\frac{1}{\log \epsilon^{-1}} u_{\epsilon, t}=\Delta u_{\epsilon}+\epsilon^{-2} u_{\epsilon}\left(1-\left|u_{\epsilon}\right|^{2}\right),
$$

the nonlinear heat (NLH) equation, or the Ginzburg-Landau equation; and

$$
i u_{\epsilon, t}=\Delta u_{\epsilon}+\epsilon^{-2} u_{\epsilon}\left(1-\left|u_{\epsilon}\right|^{2}\right),
$$

the nonlinear Schrödinger (NLS) equation, on a bounded domain $\Omega \subset \mathbb{R}^{2}$ with smooth boundary. The boundary condition is: $\left.u_{\epsilon}\right|_{\partial \Omega}=g(x)$, with $g: \partial \Omega \rightarrow \mathbb{S}^{1}$ a smooth map of degree $d>0$. The initial condition contains $d$ vortices of degree one so that the total initial energy has the asymptotic expression:

$$
\begin{aligned}
& E_{\epsilon}\left(u_{\epsilon}(0, x)\right)= \\
& \qquad \int_{\Omega} e_{\epsilon}\left(u_{\epsilon}(0, x)\right) \equiv \int_{\Omega} \frac{1}{2}\left|\nabla u_{\epsilon}\right|^{2}+\frac{\left(1-\left|u_{\epsilon}\right|^{2}\right)^{2}}{4 \epsilon^{2}}=d \pi \log \frac{1}{\epsilon}+O(1),
\end{aligned}
$$

for NLH and NLS; and

$$
E_{\epsilon}\left(u_{\epsilon}(0, x)\right)+\frac{1}{2 \log \frac{1}{\epsilon}} \int_{\Omega}\left|u_{\epsilon, t}(0, x)\right|^{2}=d \pi \log \frac{1}{\epsilon}+O(1),
$$

for NLW.

Received January 15, 1998

The first author was partially supported by NSF grant DMS-9706862.

The second author was partially supported by NSF grant DMS-9625680. 
The basic property independent of the equations is energy concentration:

$$
\frac{e_{\epsilon}\left(u_{\epsilon}\right) d x}{\pi \log \frac{1}{\epsilon}} \rightarrow \sum_{j=1}^{d} \delta_{a_{j}}, \quad \mathcal{M}(\Omega),
$$

for a sequence $\epsilon=\epsilon_{k} \downarrow 0$, where $a_{j}$ 's are distinct $d$ points inside $\Omega$, see Lin [3] and [4]. However, the dynamics of these points depends critically on the equations.

Recently, the present authors [5] formulated the conservation of linear momentum of NLS as the Euler equation:

$$
\partial_{t} p\left(u_{\epsilon}\right)=2 \operatorname{div}\left(\nabla u_{\epsilon} \otimes \nabla u_{\epsilon}\right)-\nabla P_{\epsilon},
$$

where the linear momentum $p\left(u_{\epsilon}\right)=u_{\epsilon} \wedge \nabla u_{\epsilon}$, and the pressure is:

$$
P_{\epsilon}=\left|\nabla u_{\epsilon}\right|^{2}+u_{\epsilon} \cdot \Delta u_{\epsilon}-\frac{\left|u_{\epsilon}\right|^{4}-1}{2 \epsilon^{2}} .
$$

They then projected (7) onto the locally divergence-free fields, and analyzed the resulting equation. It follows that the vortices move Lipschitz continuously on the time scale $t \sim O(1)$. Also the solution $u^{\epsilon}$ has weak limit:

$$
u_{\epsilon} \rightarrow e^{i h_{a}(x)} \prod_{j=1}^{d} \frac{x-a_{j}}{\left|x-a_{j}\right|} \equiv u_{a},
$$

away from $a_{j}$ 's in space time $L^{1}$, and that $h_{a}$ is a harmonic function on $\Omega$ satisfying $\left.h_{a, \tau}\right|_{\partial \Omega}=-\Theta_{a, \tau}+g \wedge g_{\tau}, \Theta$ the total phase of the product term in (8), and $\tau$ the tangential unit vector. It then follows from a refined energy argument that the quadratic tensor product weakly converges as:

$$
\nabla u_{\epsilon} \otimes \nabla u_{\epsilon} \rightarrow v \otimes v+\mu, \quad \mathcal{M}\left(\Omega_{a}\right),
$$

where $\mu$ is a symmetric tensorial Radon defect measure of finite mass over $\Omega$. Under the energy almost minimizing condition on the initial data:

$$
E_{\epsilon}\left(u_{\epsilon}\right)(0)=d \pi \log \frac{1}{\epsilon}+\pi W(a(0))+o(1),
$$

where $W=W(a)=W\left(a_{1}, \cdots, a_{d}\right)$ the renormalized energy, see [1], [3], it is shown in [5] that the defect measure $\mu=0$ in (9) and the vortex motion law is: $a_{j}^{\prime}=-J \nabla_{a_{j}} W, J$ being the clockwise rotation matrix. If the initial energy is an $O(1)$ amount above the minimum value (for the prescribed initial vortex locations $a(0))$, the defect measure $\mu \neq 0$, and further knowledge of its structure is necessary to deduce the law. Physically, an excessive $O(1)$ energy can allow phase (sound) waves to form and interact with vortices, while almost energy minimizing condition (10) provides only vortex self-energy $d \pi \log \frac{1}{\epsilon}$ and vortexvortex interaction energy $\pi W(a(0))$. 
For NLW, the analogous Euler equation is:

$$
\left(\frac{u_{\epsilon, t} \nabla u_{\epsilon}}{\log \epsilon^{-1}}\right)_{t}=\operatorname{div}\left(\nabla u_{\epsilon} \otimes \nabla u_{\epsilon}\right)+\nabla P_{\epsilon}
$$

with the pressure:

$$
P_{\epsilon}=\frac{u_{\epsilon, t}^{2}}{2 \log \epsilon^{-1}}-\frac{\left(1-\left|u_{\epsilon}\right|^{2}\right)^{2}}{4 \epsilon^{2}}-\frac{\left|\nabla u_{\epsilon}\right|^{2}}{2} .
$$

To analyze (11), the following energy (in)equalities are needed:

$$
\begin{aligned}
& E_{\epsilon}\left(u_{\epsilon}\right) \leq d \pi \log \frac{1}{\epsilon}+O(1), \quad \frac{1}{\log \frac{1}{\epsilon}} \int_{\Omega}\left|u_{\epsilon, t}\right|^{2} \leq C \\
& \frac{d}{d t} \int_{B_{R}} x \frac{e_{\epsilon}\left(u_{\epsilon}\right)}{\log \frac{1}{\epsilon}}=-\int_{B_{R}} \frac{u_{\epsilon, t} \cdot \nabla u}{\log \frac{1}{\epsilon}}-\frac{d}{d t} \int_{B_{R}} \frac{\left|u_{\epsilon, t}\right|^{2}}{2 \log ^{2} \frac{1}{\epsilon}}-\int_{\partial B_{R}} x \cdot u_{\epsilon, \nu} \frac{u_{\epsilon, t}}{\log \frac{1}{\epsilon}}
\end{aligned}
$$

with $B_{R} \subset \Omega$, any small ball of radius $R$. These and related energy inequalities imply the Lipschitz continuous motion of vortices, see [4]. From (12), (13) and the energy concentration, it follows that as measures:

$$
\frac{u_{\epsilon, t} \nabla u_{\epsilon}}{\pi \log \frac{1}{\epsilon}} \rightarrow-\sum_{j=1}^{d} a_{j}^{\prime}(t) \delta_{a_{j}(t)} .
$$

Moreover, the weak limit and quadratic terms also obey (8) and (9). The same energy argument [5] shows $\mu \in \mathcal{M}(\Omega)$. In addition under the energy almost minimizing condition (10), the defect measure $\mu=0$.

For NLH, energy inequalities similar to (12) hold, and the Euler equation is:

$$
\frac{d}{d t}\left(\frac{x e_{\epsilon}\left(u_{\epsilon}\right)}{\log \frac{1}{\epsilon}}\right)=-\left(\log \frac{1}{\epsilon}\right)^{-2} x\left|u_{\epsilon, \tau}\right|^{2}+\operatorname{div}\left(\nabla u_{\epsilon} \otimes \nabla u_{\epsilon}\right)+\nabla P_{\epsilon}
$$

with pressure:

$$
P_{\epsilon}=\frac{1}{\log \frac{1}{\epsilon}} x \cdot u_{\epsilon, t} \cdot \nabla u_{\epsilon}+\frac{1}{2}\left|\nabla u_{\epsilon}\right|^{2}+\frac{1}{4 \epsilon^{2}}\left(1-\left|u_{\epsilon}\right|^{2}\right)^{2} .
$$

The first term on the right hand side of (15) tends to zero in $L^{1}\left([0, T] ; L^{1}(\Omega)\right)$.

The rest of the note is organized as follows. In section 2 , we derive the vortex motion law for NLW: $a_{j}^{\prime \prime}=-\nabla_{a_{j}} W$, under the energy almost minimizing condition (10) and so $\mu=0$. The proof also works for the motion law of NLH: $a_{j}^{\prime}=-\nabla_{a_{j}} W$, which was proved earlier in Lin [3]. In this case, energy minimizing condition (10) is not needed at $t=0$. The defect measure $\mu=0$ due to energy dissipation and gradient dynamics, which imply the strong convergence of solutions away from vortices [3]. We end the note with a few remarks, comparing the three equations. 


\section{Motion law of NLW and remarks}

Let us consider a test function $\varphi \in C_{0}^{\infty}(\Omega), \varphi=n \cdot x$, for $x \in B_{R / 2}\left(a_{j}(s)\right)$, and $\varphi=0$, for $x \notin B_{R}\left(a_{j}(s)\right)$, where $R$ is a small positive number, and $n$ is a fixed unit direction. Let time $t \in[s, s+k]$ with $k$ a fixed small positive number. Multiplying $\nabla^{\perp} \varphi=\left(-\varphi_{x_{2}}, \varphi_{x_{1}}\right)$ on both sides of NLW Euler (11), integrating over $B_{R}\left(a_{j}(s)\right) \times[s, s+k]$, we get:

$$
\left.\int_{B_{R}\left(a_{j}\right)} \frac{u_{\epsilon, t} \nabla u_{\epsilon}}{\log \epsilon^{-1}} \cdot \nabla^{\perp} \varphi\right|_{s} ^{s+k}=\int_{s}^{s+k} d t \int_{B_{R}\left(a_{j}\right)} \operatorname{div}\left(\nabla u_{\epsilon} \otimes \nabla u_{\epsilon}\right) \cdot \nabla^{\perp} \varphi .
$$

Passing $\epsilon \downarrow 0$, we find using basic facts of section 1 that:

$$
\begin{aligned}
\left.\pi a_{j}^{\prime}(t) \cdot n^{\perp}\right|_{s} ^{s+k} & =\lim _{\epsilon \downarrow 0} \int_{s}^{s+k} d t \int_{B_{R}\left(a_{j}\right)}\left(\nabla u_{\epsilon} \otimes \nabla u_{\epsilon}\right): \nabla \nabla^{\perp} \varphi \\
& =\lim _{\epsilon \downarrow 0} \int_{s}^{s+k} d t \int_{B_{R}\left(a_{j}\right) \backslash B_{R / 2}\left(a_{j}\right)}\left(\nabla u_{\epsilon} \otimes \nabla u_{\epsilon}\right): \nabla \nabla^{\perp} \varphi \\
& =\int_{s}^{s+k} d t \int_{B_{R}\left(a_{j}\right) \backslash B_{R / 2}\left(a_{j}\right)}(v \otimes v+\mu): \nabla \nabla^{\perp} \varphi \\
& =\int_{s}^{s+k} d t \int_{B_{R}\left(a_{j}\right)}(v \otimes v+\mu): \nabla \nabla^{\perp} \varphi \\
& =\int_{s}^{s+k} d t \int_{B_{R}\left(a_{j}\right)} v \otimes v: \nabla \nabla^{\perp} \varphi
\end{aligned}
$$

where in the last equality we have used the almost energy minimizing assumption (10) to exclude $\mu$. We also see that in general $\mu$ does not contribute if $\operatorname{div} \mu=$ $-\nabla P_{\mu}$, globally on $\Omega$ for a distribution $P_{\mu}$.

It follows via integration by parts that:

$$
\begin{aligned}
\left.\pi a_{j}^{\prime}(t) \cdot n^{\perp}\right|_{s} ^{s+k} & =\int_{s}^{s+k} d t \int_{B_{R}\left(a_{j}(s)\right) \backslash B_{R / 2}\left(a_{j}(s)\right)}(v \otimes v): \nabla \nabla^{\perp} \varphi \\
& =-\int_{s}^{s+k} d t \int_{B_{R}\left(a_{j}(s)\right) \backslash B_{R / 2}\left(a_{j}(s)\right)} v \cdot \nabla v \cdot \nabla^{\perp} \varphi \\
& +\int_{s}^{s+k} d t \int_{\partial B_{R / 2}\left(a_{j}(s)\right)}(v \otimes v):\left(\nu \otimes n^{\perp}\right) \\
& =-\int_{s}^{s+k} d t \int_{\partial B_{R / 2}\left(a_{j}(s)\right)}\left(v \cdot \nabla v \cdot \nu^{\perp}\right)(n \cdot x) \\
& +\int_{s}^{s+k} d t \int_{\partial B_{R / 2}\left(a_{j}(s)\right)}(v \otimes v):\left(\nu \otimes n^{\perp}\right) .
\end{aligned}
$$


Dividing (3) by $k$ and letting $k \downarrow 0$, we obtain:

$$
\begin{aligned}
& \pi a_{j}^{\prime \prime}(s) \cdot n^{\perp}= \\
& \quad-\int_{\partial B_{R / 2}\left(a_{j}(s)\right)}\left(v \cdot \nabla v \cdot \nu^{\perp}\right)(n \cdot x)+\int_{\partial B_{R / 2}\left(a_{j}(s)\right)}(v \otimes v):\left(\nu \otimes n^{\perp}\right) .
\end{aligned}
$$

Letting $n=(1,0)$, and $a_{j}=\left(\xi_{j}, \eta_{j}\right)$, we calculate with the explicit expression for $v=\left(v_{1}, v_{2}\right)$ as $x \sim a_{j}: v_{1}=H_{j, \xi}-r^{-1} \sin \theta, \quad v_{2}=H_{j, \eta}+r^{-1} \cos \theta$, where $H_{j}$ is the smooth part of the vortex phase $\Theta_{a}+h_{a}$ at $a_{j},(r, \theta)$ is the local polar coordinate. We find that each term on the right hand side of (4) contributes $-\pi H_{j, \xi}$, and so: $\pi \eta^{\prime \prime}(s)=-2 \pi H_{j, \xi}$. Similarly with $n=(0,1)$, we find: $-\pi \xi^{\prime \prime}(s)=-2 \pi H_{j, \eta}$. By the conjugation relation between $H_{j}$ and $W$, [1], we have: $J a_{j}^{\prime \prime}=-2 \nabla H_{j}=-J \nabla_{a_{j}} W$, where $J$ is the $\pi / 2$ clockwise rotation matrix. The vortex motion law: $a_{j}^{\prime \prime}=-\nabla_{a_{j}} W$ follows. For NLH, the argument is the same except the left hand side of the motion law is $a_{j}^{\prime}$ due to (6) and (15). We have completed the proof of the vortex motion laws of NLW and NLH.

A few remarks are in order. The left hand sides of the Euler-like equations of NLW and NLH concentrate. Hence away from vortices, we have essentially the steady state Euler equation. The pressure $P_{\epsilon}$ is in $L^{1}\left([0, T] ; L^{1}\left(\Omega_{a}\right)\right), \Omega_{a}=$ $\left\{\Omega \backslash\left(a_{1}, \cdots, a_{d}\right)\right\}$. Letting $P^{\prime}$ be the weak $L^{1}$ limit of $P_{\epsilon}$, and passing $\epsilon \downarrow 0$ on $\Omega_{a}$, we have from (11) or (15) that:

$$
\operatorname{div}(v \otimes v)+\nabla P=0, \quad \operatorname{div} v=0, \quad P=P_{\mu}+P^{\prime},
$$

in the sense of distribution, where $P$ is the total pressure. It follows that $\operatorname{div}(v$. $\nabla v)=-\Delta P$, and $P$ is smooth on $\Omega_{a}$. Solving for $P$ near each vortex $a_{j}$ gives the general expression of:

$$
P=\frac{1}{2} r^{-2}+\alpha r^{-1} \cos \theta+\beta r^{-1} \sin \theta+\gamma \log r+\cdots,
$$

where $\cdots$ are either higher spherical harmonic terms with frequencies at least two or bounded regular terms, $r=\left|x-a_{j}\right|$. Calculating circulation from (5) gives to leading order:

$$
\pi \beta=\int_{\partial B_{r}\left(a_{j}\right)} \xi P_{\theta}=-\oint_{\partial B_{r}\left(a_{j}\right)} \xi v \cdot \nabla v d \vec{l}=\pi H_{j, \xi}\left(a_{j}\right) .
$$

So $\beta=H_{j, \xi}\left(a_{j}\right)$. Similarly, $\alpha=-H_{j, \eta}\left(a_{j}\right)$.

In contrast, the NLS pressure $P_{\epsilon}$ is not controled by energy inequality and not known to stay in $L^{1}$ as $\epsilon \downarrow 0$. Also the left hand side $p\left(u_{\epsilon}\right)$ converges to a smooth function away from vortices, and $p\left(u_{\epsilon}\right)$ itself does not concentrate. Hence it is convenient to project the Euler equation to divergence free fields and calculate circulations [5]. The limiting equation is the time dependent Euler. It turns out that in the asymptotic expression for the limiting total pressure $P, \alpha$ and $\beta$ are undertermined in general. The energy almost minimizing condition (10) of course implies that $\alpha=\beta=0$ in this case. 
Last but not the least, we remark that the regime where defect measure forms is beyond the reach of early formal asymptotic derivations, see [6], [7], among others. From our analysis, we gain new understanding that the motion law holds as suggested in formal derivations if the divergence of the finite mass defect measure is a global pressure gradient or is the defect measure if supported away from the vicinities of vortices. In most early formal works, the connection with the field aspect of the problem, namely pressure, is not exploited, much less the fluid dynamic perspective of all three scalar field equations. Analogous defect measure is well-known to play a significant role in the study of weak solutions of two-dimensional incompressible Euler equation in fluid dynamics [2].

\section{Acknowledgement}

We thank R. Jerrard for his comments on our early manuscript.

\section{References}

1. T. Bethuel, H. Brezis, and F. Helein, Ginzburg-Landau vortices, Birkhauser, Boston, 1994.

2. R. DiPerna and A. Majda, Reduced Hausdorff dimension and concentration-cancellation for 2-D incompressible flow, J. Amer. Math. Soc. 1, (1988), 59-95.

3. F.-H. Lin, Some dynamical properties of Ginzburg-Landau vortices, Comm. Pure Appl. Math, XLIX(1996), pp. 323-359; and remarks on this paper, Ibid, XLIX(1996), pp. 361364.

4. __ Vortex dynamics for the nonlinear wave equations, Comm. Pure Appl. Math, (to appear).

5. F.-H. Lin and J. Xin, On the incompressible fluid limit and the vortex motion law of the nonlinear Schrödinger equation, Comm. Math. Phys., (to appear).

6. J. Neu, Vortices in the complex scalar fields, Physica D 43 (1990), 385-406.

7. __ Vortex dynamics of the nonlinear wave equation, Physica D 43 (1990), 407-420.

Courant Institute, New York University, 251 Mercer Street, NY, NY 10012

E-mail address: linf@math1.cims.nyu.edu

Dept. of Math., University of Arizona, Tucson, AZ 85721

E-mail address: xin@math.arizona.edu 